\title{
RNA-based regulation in type I toxin-antitoxin systems and its implication for bacterial persistence
}

\author{
Bork A. Berghoff ${ }^{1}$ E. Gerhart H. Wagner ${ }^{2}(0)$
}

Received: 15 May 2017 / Revised: 19 May 2017 / Accepted: 22 May 2017 / Published online: 30 May 2017

(C) The Author(s) 2017. This article is an open access publication

\begin{abstract}
Bacterial dormancy is a valuable survival strategy upon challenging environmental conditions. Dormant cells tolerate the consequences of high stress levels and may re-populate the environment upon return to favorable conditions. Antibiotic-tolerant bacteria-termed persisters-regularly cause relapsing infections, increase the likelihood of antibiotic resistance, and, therefore, earn increasing attention. Their generation often depends on toxins from chromosomal toxin-antitoxin systems. Here, we review recent insights concerning RNA-based control of toxin synthesis, and discuss possible implications for persister generation.
\end{abstract}

Keywords Toxin-antitoxin · Antisense RNA $\cdot 5^{\prime}$ UTR structure $\cdot$ Persistence $\cdot$ Depolarization $\cdot$ SOS response

\section{Introduction}

Every organism's future is unwritten and to a large extent unpredictable. We-as human beings-are aware of this unpleasant fact and try to safeguard ourselves by sanitary and monetary protection. Even though simple organisms

Communicated by M. Kupiec.

Bork A. Berghoff

Bork.A.Berghoff@mikro.bio.uni-giessen.de

E. Gerhart H. Wagner

gerhart.wagner@icm.uu.se

1 Institut für Mikrobiologie und Molekularbiologie, Justus-Liebig-Universität, 35392 Giessen, Germany

2 Department of Cell and Molecular Biology, Uppsala University, 75124 Uppsala, Sweden like bacteria are not "aware" of the inevitable risks of life, they have inherited genetic programs that have ensured their survival in the past and will do so in the future. Generating phenotypic heterogeneity in a clonal population of bacteria is considered a successful survival strategy, often referred to as bet-hedging (Veening et al. 2008). For example, most bacteria generate subpopulations of nongrowing (i.e., dormant) cells that can withstand unfavorable environmental conditions (Lennon and Jones 2011). According to the "microbial scout" hypothesis, cells leave dormancy stochastically to sample the environment (Buerger et al. 2012; Sturm and Dworkin 2015). If conditions are favorable, these pioneering cells can re-populate the environment. Even the smallest subpopulation that rides out a catastrophe can ensure continuity of the bacterial species as such. In line with this concept, pathogenic bacteria generate multidrug-tolerant phenotypic variants that have been denoted persisters due to their ability to survive antibiotic treatment (Bigger 1944). Persisters regularly cause relapsing infections and are considered a major risk to public health (Lewis 2010). In contrast to resistant cells, persisters are unable to multiply in the presence of antibiotics, but rather reside in a dormant state which renders them tolerant towards the action of most antibiotics. Multiple pathways by which persisters arise have been described, most often ultimately resulting in slowed down growth via corruption of essential cellular processes. For example, persistence can be triggered by ATP depletion (Conlon et al. 2016; Shan et al. 2017), nutrient shifts, and metabolic perturbations (Amato et al. 2013; Amato and Brynildsen 2014; Radzikowski et al. 2016), stochastic induction of (p)ppGpp (Maisonneuve et al. 2013; Germain et al. 2015), or indole-activated stress responses (Vega et al. 2012). Distinct pathways might be either essential or rather negligible for persister 
formation, depending on the experimental/environmental conditions. For example, it was recently challenged whether (p)ppGpp-activated pathways are the dominant source of persister cells (Chowdhury et al. 2016; Shan et al. 2017), and clearly, more experiments are needed to understand the complex nature of persister formation. A recurrent scheme for inducing the persistent state involves toxins from chromosomal toxin-antitoxin (TA) systems (e.g., Dörr et al. 2010; Kim and Wood 2010; Maisonneuve et al. 2011). In unstressed cells, antitoxins normally inhibit either translation or activity of their toxin counterparts. However, when stress occurs, the inhibiting effect is released and cellular processes are impeded by the action of one or several toxins. The different TA systems are classified according to the specific mechanism by which the antitoxin inhibits the toxin directly, or its synthesis. In total, six different TA system types have been described so far (reviewed in Page and Peti 2016). Translational repression of toxin mRNA by an antisense RNA (type I) and inhibition of toxin activity by an antitoxin via protein-protein interaction (type II) are the predominant mechanisms. The first persistencerelated toxin gene was hipA in Escherichia coli (Moyed and Bertrand 1983). HipA belongs to the type II TA system HipAB. Its mode of action was recently deciphered: HipA phosphorylates glutamyl-tRNA synthetase, causing uncharged tRNA accumulation, thereby triggering the synthesis of the alarmone (p)ppGpp (Germain et al. 2013, 2015; Kaspy et al. 2013). Accumulation of (p) ppGpp results in activation of Lon protease, which, in turn, activates several toxins from type II TA systems via degradation of the cognate antitoxins. Most of the Lonactivated toxins are RNA endonucleases that corrupt translation, induce growth arrest, and lead to persister formation (Maisonneuve et al. 2011, 2013). Moreover, some RNA endonucleases were reported to impact stress responses and biofilm formation (Wang and Wood 2011), or to attack phage mRNAs to obstruct phage propagation (Otsuka 2016). Type II TA systems have intensively been studied with regard to the particular mode of toxin action, the regulatory interplay between the toxin-antitoxin partners, and the implications for persister formation (see Gerdes and Maisonneuve 2012; Maisonneuve and Gerdes 2014; Page and Peti 2016; Rocker and Meinhart 2016 for recent reviews). In this review, we will discuss the peculiarities of type I TA systems. Most toxins of type I TA systems are small hydrophobic peptides $(<60$ amino acids), some of which target the inner membrane to cause depolarization and ATP depletion. This entails inhibition of major cellular processes which is believed to induce persister formation (Unoson and Wagner 2008; Dörr et al. 2010; Verstraeten et al. 2015; Berghoff et al. 2017). Based on current knowledge, we will present new concepts regarding regulation of type I TA systems and bacterial persistence.

\section{How to keep toxins at bay: the hok/sok paradigm}

Type I toxin-antitoxin systems were initially discovered in bacterial plasmids as post-segregational killing (PSK) systems. Such loci, exemplified by its founding member hok/sok on plasmid R1, confer stable plasmid maintenance by killing cells that have lost the plasmid (Gerdes et al. 1986; Weaver and Tritle 1994). The unstable antitoxin RNA is rapidly purged from plasmid-free progeny cells, leading to uninhibited translation of the stable toxin mRNA; the toxin causes cell death through membrane damage. Killing makes sense from the point of view of plasmid maintenance, but for chromosomally encoded TA systems, less is known on whether toxicity plays out as killing or growth arrest/retardation. Clearly, in plasmid-containing cells, toxicity must be tightly controlled. The same holds true for chromosomally encoded type I TA systems which were initially identified as homologues of their plasmid counterparts like hok/sok (Gerdes et al. 1986). The E. coli K-12 genome encodes five hok/sok homologues (Pedersen and Gerdes 1999), and a recent study implicates one of these systems (hokB/ sokB) in membrane depolarization and persister formation (Verstraeten et al. 2015). Regulation of hok expression has thoroughly been studied in the plasmid-borne system (reviewed in Gerdes and Wagner 2007), and the same regulatory principles are expected to apply to the chromosomal ones. Translation of hok ("host killing") depends on translation of the overlapping upstream reading frame mok ("modulation of killing"). The primary full-length mok-hok transcript (398 nt) is stable and translationally inert due to structural sequestration. However, slow processing by RNase II and polyribonucleotide nucleotidyltransferase (PNPase) removes 39 nt from the $3^{\prime}$ end and induces structural rearrangements, resulting in a translationally active mRNA (361 nt; Fig. 1). In plasmid-containing cells, the RNA antitoxin Sok ("suppressor of killing") binds to an accessible U-turn structure in the target loop of the 361-nt-long transcript and represses $m o k$, and consequently hok translation (Fig. 1). The RNA heteroduplex is cleaved by RNase III and then decays. Thus, inhibition of Hok translation depends on two regulatory RNA elements: an inhibitory secondary structure in the inert mok-hok transcript and the antitoxin Sok. This is reminiscent of other chromosomal type I TA systems found in E. coli, and two-layer control might represent a general regulatory feature of some of these systems. 

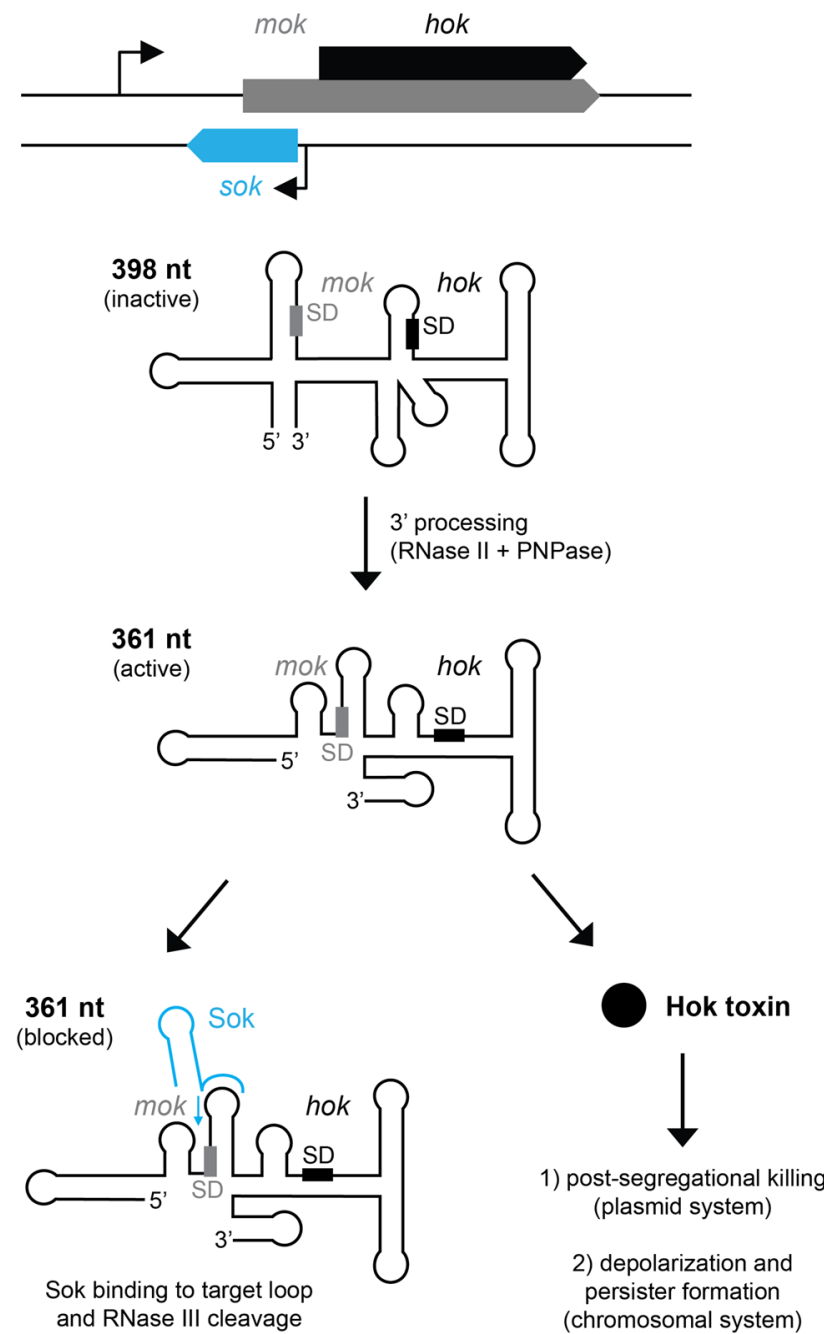

Fig. 1 Synthesis of Hok toxins is controlled by two regulatory RNA elements. The arrangement of the hok/sok gene locus is depicted in the upper part of the figure. Arrows indicate promoters. The primary mok-hok mRNA (398 nt) is translationally inert due to inhibitory secondary structures. $3^{\prime}$ processing generates a translationally active mRNA (361 nt), that is either inhibited by the RNA antitoxin Sok or translated into Hok toxin. Sok binding initiates at the target loop, and the RNA duplex that subsequently forms (indicated by blue arrow) is cleaved by RNase III. Plasmid-borne systems are implicated in postsegregational killing, and chromosomal systems contribute to bacterial persistence. $S D$ Shine-Dalgarno sequence. Figure based on Gerdes and Wagner (2007). See text for details

\section{SOS induced toxins: TisB and DinQ}

The chromosomally encoded type I TA systems tisB/ istR- 1 and $\operatorname{din} Q / \operatorname{agr} B$ in $E$. coli are implicated in persister formation and survival upon DNA damage, respectively (Dörr et al. 2010; Weel-Sneve et al. 2013; Berghoff et al. 2017). In both systems, the toxin and antitoxin genes are transcribed divergently in a non-overlapping fashion. While the antitoxins are constitutively expressed, transcription of the toxin genes is controlled by LexA, the master regulator of the response to DNA damage (SOS response) (Fig. 2). Under non-stress conditions, LexA binds to the so-called LexA boxes within the promoter region of SOS genes and represses transcription. LexA box sequences are classified by their "heterology index" (HI), indicating how tightly the LexA repressor binds to the particular sequence: a low $\mathrm{HI}$ indicates tight repression (Lewis et al. 1994). It makes sense that transcriptional control by LexA repression is complemented by inhibition at the post-transcriptional level, because transcriptional off-states are intrinsically difficult to obtain (Golding et al. 2005; Levine and Hwa 2008). Indeed, leaky transcription is observed for both tisB (Berghoff et al. 2017) and $\operatorname{din} Q$ (Weel-Sneve et al. 2013), even though both genes have comparably low HI indices of 1.81 and 3.92, respectively (Courcelle et al. 2001). Maintaining a stable off state during normal growth involves two RNA elements. In the case of tisB, one is an inhibitory $5^{\prime}$ UTR structure that masks a ribosome standby site (RSS) in the +1 transcript to prevent translation (Darfeuille et al. 2007). A processing event generates the translationally active $+42 \mathrm{mRNA}$, in which the RSS is accessible for ribosome preloading (Fig. 2a). A scarless chromosomal deletion of the first 41 nucleotides of $t i s B$ mRNA $(\Delta 1-41)$ shows leaky expression of the translationally active $+42 \mathrm{mRNA}$, and consequently, inappropriate TisB synthesis and depolarization under conditions of low DNA damage (Berghoff et al. 2017). Since binding of the antitoxin IstR- 1 to the +42 mRNA triggers RNase III cleavage and thereby removes +42 transcripts, the effect on depolarization is potentiated when IstR-1 is simultaneously deleted. These results demonstrate that an additional regulatory element (the inhibitory $5^{\prime}$ UTR structure) acts to complement the antitoxin RNA for tight control of toxin expression.

The $\operatorname{din} Q / a g r B$ system shares many similarities with tisB/istR-1 with respect to post-transcriptional regulation: the $\operatorname{din} Q+1$ transcript is translationally inactive and has to be processed at its $5^{\prime}$ end to produce the translationally active +44 transcript. Translation of +44 mRNA is repressed by binding of the antitoxin AgrB (Weel-Sneve et al. 2013; Kristiansen et al. 2016) (Fig. 2b). There are, however, differences between the $\operatorname{din} Q / \operatorname{agr} B$ and tisB/ istR-1 systems: (1) translation of $\operatorname{din} Q+44$ mRNA does not rely on ribosome standby, (2) AgrB does not directly compete with ribosome binding, but rather induces structural rearrangements to sequester the Shine-Dalgarno (SD) sequence, and (3) the primary function of DinQ toxin might not be depolarization of the inner membrane (Fig. 2b). Although plasmid-borne overexpression of DinQ causes depolarization (Weel-Sneve et al. 2013), deletion of $\operatorname{din} Q$ does not affect depolarization upon ciprofloxacin treatment, and yet, persistence is impeded (our unpublished 
a
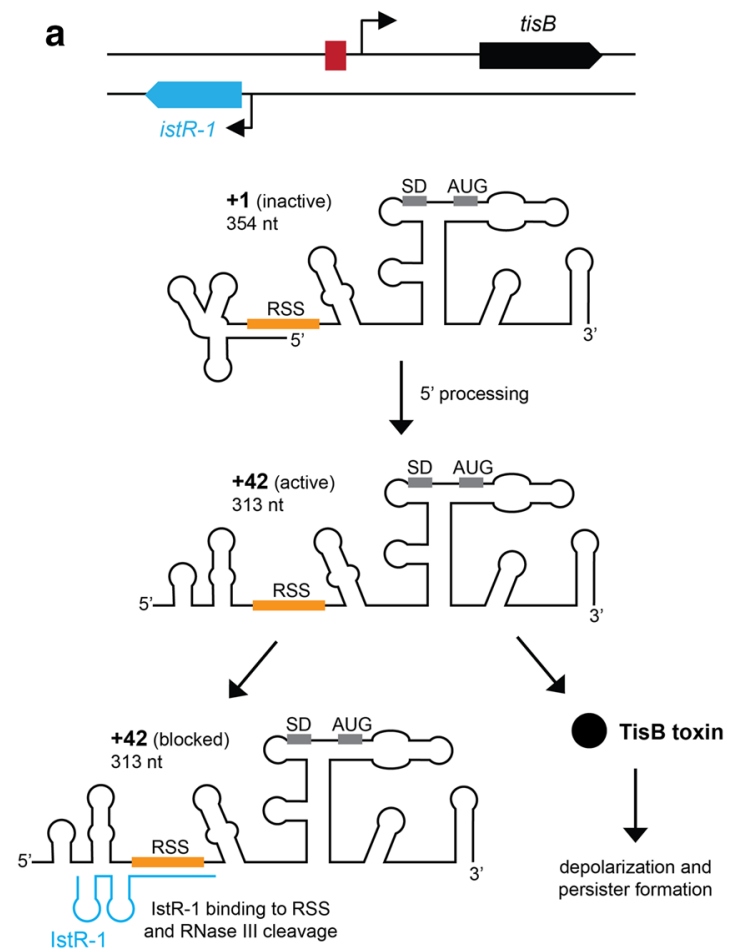

Fig. 2 RNA-based regulation of the SOS-responsive toxins TisB and DinQ. The loci for tisB/istR-1 (a) and $\operatorname{din} Q / \operatorname{ag} r A B(\mathbf{b})$ are depicted in the upper part of the figure. Arrows indicate promoters and red boxes represent binding sites for LexA. In both cases, primary mRNAs $(+1)$ are translationally inert due to secondary structures that prevent ribosome binding. $5^{\prime}$ processing generates translationally active mRNAs

data). By contrast, deletion of tisB abolishes depolarization, and TisB, therefore, appears to be the main factor for membrane depolarization under SOS conditions (Berghoff et al. 2017). DinQ may primarily regulate nucleoid compaction during repair of DNA lesions, since moderate overexpression of DinQ (by agrB deletion) leads to an extended period of nucleoid compaction (Weel-Sneve et al. 2013). The reduced survival of the $\operatorname{agrB}$ deletion strain upon UV stress further demonstrates that DinQ levels need to be tightly controlled, and that the regulatory RNA elements play important roles in this regard.

\section{Further toxin mRNAs with regulatory elements in their $5^{\prime}$ UTRs: zorO and shoB}

Besides tisB/istR- 1 and $\operatorname{din} Q / \operatorname{agr} B$, two additional type I TA systems display a divergent gene orientation and antitoxin RNA-binding sites far upstream of the ribosomebinding site (RBS) of the toxin mRNA. In E. coli $\mathrm{O} 157: \mathrm{H} 7$ (EHEC), the zorO-orzO locus encodes the ZorO toxin and the antisense RNA OrzO. OrzO counteracts ZorO toxicity by base pairing to the mRNA (Wen et al. 2014). The b
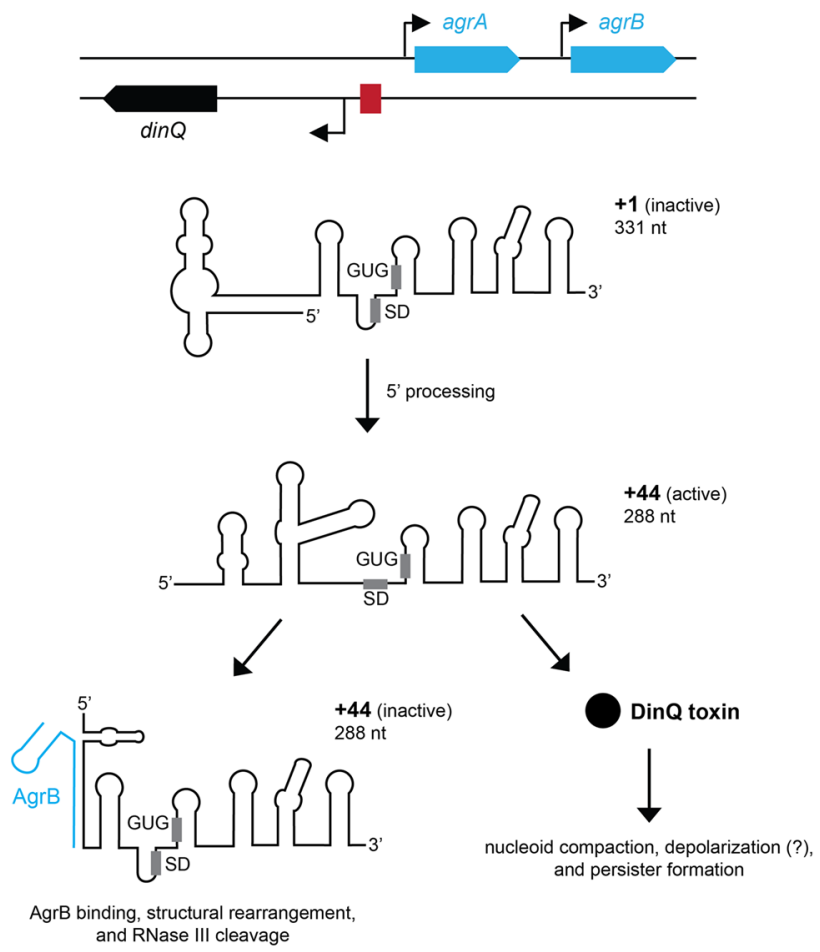

(tisB +42 or $\operatorname{din} Q+44$ ) with accessible sites for ribosome loading. The active mRNAs are either inhibited by their cognate RNA antitoxins, or translated into toxin. Both TisB and DinQ are implicated in persister formation under SOS conditions. AUG/GUG: start codon; $S D$ Shine-Dalgarno sequence, $R S S$ ribosome standby site. See text for details

primary zorO mRNA supports little translation due to sequestration of the RBS in a stem. As in the case of tisB, $5^{\prime}$ processing generates a translationally active transcript: removal of the $5^{\prime}$-most 28 nucleotides causes a structure change, so that ribosome preloading at a single-stranded RSS can occur (Wen et al. 2016). The OrzO RNA competes with ribosome standby and triggers RNase III cleavage. ZorO is a small hydrophobic peptide ( 29 amino acids) that may contribute to regulating cellular growth in response to nutrient shifts (Wen et al. 2014).

The $s h o B-o h s C$ locus was identified in a screen for small RNAs in E. coli (Kawano et al. 2005) and subsequently shown to constitute a bona fide type I TA pair: ShoB toxicity can be abolished by OhsC expression (Fozo et al. 2008). Full-length ( $320 \mathrm{nt})$ and $5^{\prime}$ processed $(\sim 280 \mathrm{nt})$ shoB transcripts were identified, but again, only the processed mRNA was translationally active (Fozo et al. 2008). The regulatory elements within $s h o B$ mRNA have not yet been studied in detail, but likely involves secondary structures that inhibit translation and need to be resolved by $5^{\prime}$ processing. The small hydrophobic ShoB toxin (26 amino acids) causes depolarization upon overexpression (Fozo et al. 2008). It will be exciting to see whether 
and-if so- - under which conditions ShoB and ZorO contribute to bacterial persistence, and whether depolarization is involved.

\section{Two-layer control of toxin translation blurs the definition of antitoxins}

Why are many type I toxin mRNAs endowed with inhibitory RNA elements in their $5^{\prime}$ UTRs? One shared property is that, as for hok and tisB mRNAs, translation is not coupled to transcription. That is, mRNAs first accumulate in a translationally inactive form, and toxin synthesis only occurs after processing. Here, the RNA antitoxin alone is not sufficient to tightly block toxin expression, as supported by recent results with the tisB/istR-1 system. Deletion of the tisB $5^{\prime}$ UTR structure triggers depolarization even without strong SOS induction and in the presence of the antitoxin IstR-1 (Berghoff et al. 2017). Most intriguingly, persister levels of $\Delta i s t R$ and $\Delta 1-41$ single deletion strains were increased by 4 - and 11-fold, respectively, in comparison to the wild type upon ciprofloxacin treatment (Berghoff et al. 2017). The $5^{\prime}$ UTR structure seems, in our experimental setting, to be more important for controlling TisBdependent persister formation than the antitoxin RNA. This can be interpreted as control by two antitoxin elements: the intrinsic antitoxin (toxin mRNA $5^{\prime}$ structure) and the extrinsic antitoxin (antisense RNA). Importantly, the "two antitoxins" act sequentially, which causes a delay in toxin synthesis. As long as the intrinsic $5^{\prime}$ UTR structure is present in the toxin mRNA, no translation occurs, and binding of the antisense RNA only plays a minor role. If the $5^{\prime}$ UTR structure is resolved by processing, the antisense RNA takes over, and mRNA decay irreversibly removes translationally active transcripts from the cell. The frequency of processing and the abundance of extrinsic RNA antitoxin, therefore, determine whether and when toxin is produced. Consequently, using "two antitoxins" for regulation generates a continuum of toxin-producing cells in terms of time and toxin abundance. This phenotypic heterogeneity likely provides an adequate rate of persister cell formation when stress occurs. Some cells may quickly produce high amounts of toxins and immediately enter the persistent state, aiding the survival of the population in case of fatal stress. Other cells may instead continue to grow first and turn into persisters later, which might be beneficial in case of mild but enduring stress. Hypothetically, upon return to non-stress conditions, the slow responder cells might not enter the persistent state at all and instead proliferate.

When wild-type $E$. coli cells were pre-treated with low ciprofloxacin concentrations $(1 \times \mathrm{MIC}$, minimal inhibitory concentration) and subsequently challenged with a high dose of ciprofloxacin $(100 \times$ MIC), persister levels were enhanced by $\sim$ tenfold compared to samples without pre-treatment (Berghoff et al. 2017). Importantly, deleting "both antitoxins" in the tisB/istR-1 system $(\Delta 1-41 \Delta i s t R)$ causes a strong increase in persister levels upon ciprofloxacin treatment ( $>100$-fold compared to the wild type), irrespective of pre-treatment. The TA system behaves as if it changed from an inducible (wild-type situation) to a stochastic mode (by deletion of both regulatory RNA elements). Assuming that the wild-type situation confers maximal fitness to $E$. coli in its natural habitats, our observations suggest two main conclusions: (1) type I TA systems, e.g., tisB/istR-1, are "built" to be silent under nonstress conditions, and (2) tight regulation by two antitoxin elements provides an adequate fraction of persisters upon environmental stress. A similar rationale would likely also apply to $h o k B / \operatorname{sokB}, \operatorname{din} Q / \operatorname{agrB}$, zorO/orzO, and shoB/ ohs $C$. Competition experiments between wild-type and antitoxin-deletion strains with regard to persistence might answer these questions in the future.

Acknowledgements B. A. B. was funded by fellowships from the European Molecular Biology Organization (EMBO, ALTF 62-2012) and the German Research Foundation (DFG, BE 5210/1-1 and BE 5210/2-1). E. G.H. W. was supported by the Swedish Research Council. We thank Erik Holmqvist for critical reading of the manuscript.

Open Access This article is distributed under the terms of the Creative Commons Attribution 4.0 International License (http://creativecommons.org/licenses/by/4.0/), which permits unrestricted use, distribution, and reproduction in any medium, provided you give appropriate credit to the original author(s) and the source, provide a link to the Creative Commons license, and indicate if changes were made.

\section{References}

Amato SM, Brynildsen MP (2014) Nutrient transitions are a source of persisters in Escherichia coli biofilms. PLoS One 9(3):e93110. doi:10.1371/journal.pone.0093110

Amato SM, Orman MA, Brynildsen MP (2013) Metabolic control of persister formation in Escherichia coli. Mol Cell 50:475-487. doi:10.1016/j.molcel.2013.04.002

Berghoff BA, Hoekzema M, Aulbach L, Wagner EGH (2017) Two regulatory RNA elements affect TisB-dependent depolarization and persister formation. Mol Microbiol 103:1020-1033. doi:10.1111/mmi.13607

Bigger JW (1944) The bactericidal action of penicillin on Staphylococcus pyogenes. Ir J Med Sci 19:553-568. doi:10.1007/ BF02948386

Buerger S, Spoering A, Gavrish E et al (2012) Microbial scout hypothesis, stochastic exit from dormancy, and the nature of slow growers. Appl Environ Microbiol 78:3221-3228. doi:10.1128/ AEM.07307-11

Chowdhury N, Kwan BW, Wood TK (2016) Persistence increases in the absence of the alarmone guanosine tetraphosphate by reducing cell growth. Sci Rep 6:20519. doi:10.1038/srep20519

Conlon BP, Rowe SE, Gandt AB et al (2016) Persister formation in Staphylococcus aureus is associated with ATP depletion. Nat Microbiol 1:16051. doi:10.1038/nmicrobiol.2016.51 
Courcelle J, Khodursky A, Peter B et al (2001) Comparative gene expression profiles following UV exposure in wild-type and SOS-deficient Escherichia coli. Genetics 158:41-64

Darfeuille F, Unoson C, Vogel J, Wagner EGH (2007) An antisense RNA inhibits translation by competing with standby ribosomes. Mol Cell 26:381-392. doi:10.1016/j.molcel.2007.04.003

Dörr T, Vulic M, Lewis K (2010) Ciprofloxacin causes persister formation by inducing the TisB toxin in Escherichia coli. PLoS Biol 8:e1000317. doi:10.1371/journal.pbio.1000317

Fozo EM, Kawano M, Fontaine F et al (2008) Repression of small toxic protein synthesis by the Sib and OhsC small RNAs. Mol Microbiol 70:1076-1093. doi:10.1111/j.1365-2958.2008.06394.x

Gerdes K, Maisonneuve E (2012) Bacterial persistence and toxinantitoxin loci. Annu Rev Microbiol 66:103-123. doi:10.1146/ annurev-micro-092611-150159

Gerdes K, Wagner EGH (2007) RNA antitoxins. Curr Opin Microbiol 10:117-124

Gerdes K, Rasmussen PB, Molin S (1986) Unique type of plasmid maintenance function: postsegregational killing of plasmid-free cells. Proc Natl Acad Sci USA 83:3116-3120

Germain E, Castro-Roa D, Zenkin N, Gerdes K (2013) Molecular mechanism of bacterial persistence by HipA. Mol Cell 52:248 254. doi:10.1016/j.molcel.2013.08.045

Germain E, Roghanian M, Gerdes K, Maisonneuve E (2015) Stochastic induction of persister cells by HipA through (p)ppGppmediated activation of mRNA endonucleases. Proc Natl Acad Sci USA 112:5171-5176. doi:10.1073/pnas.1423536112

Golding I, Paulsson J, Zawilski SM, Cox EC (2005) Real-time kinetics of gene activity in individual bacteria. Cell 123:1025-1036. doi:10.1016/j.cell.2005.09.031

Kaspy I, Rotem E, Weiss N et al (2013) HipA-mediated antibiotic persistence via phosphorylation of the glutamyl-tRNA-synthetase. Nat Commun 4:3001. doi:10.1038/ncomms4001

Kawano M, Reynolds AA, Miranda-Rios J, Storz G (2005) Detection of 5'- and 3'-UTR-derived small RNAs and cis-encoded antisense RNAs in Escherichia coli. Nucleic Acids Res 33:1040 1050. doi:10.1093/nar/gki256

Kim Y, Wood TK (2010) Toxins Hha and CspD and small RNA regulator Hfq are involved in persister cell formation through MqsR in Escherichia coli. Biochem Biophys Res Commun 391:209213. doi:10.1016/j.bbrc.2009.11.033

Kristiansen KI, Weel-Sneve R, Booth JA, Bjørås M (2016) Mutually exclusive RNA secondary structures regulate translation initiation of DinQ in Escherichia coli. RNA 22:1739-1749. doi:10.1261/rna.058461.116

Lennon JT, Jones SE (2011) Microbial seed banks: the ecological and evolutionary implications of dormancy. Nat Rev Microbiol 9:119-130. doi:10.1038/nrmicro2504

Levine E, Hwa T (2008) Small RNAs establish gene expression thresholds. Curr Opin Microbiol 11:574-579

Lewis K (2010) Persister cells. Annu Rev Microbiol 64:357-372. doi:10.1146/annurev.micro.112408.134306

Lewis L, Harlow GR, Gregg-Jolly LA, Mount DW (1994) Identification of high affinity binding sites for LexA which define new DNA damage-inducible genes in Escherichia coli. J Mol Biol 241:507-523. doi:10.1006/jmbi.1994.1528

Maisonneuve E, Gerdes K (2014) Molecular mechanisms underlying bacterial persisters. Cell 157:539-548
Maisonneuve E, Shakespeare LJ, Jørgensen MG, Gerdes K (2011) Bacterial persistence by RNA endonucleases. Proc Natl Acad Sci USA 108:13206-13211. doi:10.1073/pnas.1100186108

Maisonneuve E, Castro-Camargo M, Gerdes K (2013) (p)ppGpp controls bacterial persistence by stochastic induction of toxin-antitoxin activity. Cell 154:1140-1150. doi:10.1016/j. cell.2013.07.048

Moyed HS, Bertrand KP (1983) hipA, a newly recognized gene of Escherichia coli K-12 that affects frequency of persistence after inhibition of murein synthesis. J Bacteriol 155:768-775

Otsuka Y (2016) Prokaryotic toxin-antitoxin systems: novel regulations of the toxins. Curr Genet. doi:10.1007/s00294-015-0557-z

Page R, Peti W (2016) Toxin-antitoxin systems in bacterial growth arrest and persistence. Nat Chem Biol 12:208-214. doi:10.1038/ nchembio. 2044

Pedersen K, Gerdes K (1999) Multiple hok genes on the chromosome of Escherichia coli. Mol Microbiol 32:1090-1102

Radzikowski JL, Vedelaar S, Siegel D et al (2016) Bacterial persistence is an active $\sigma \mathrm{S}$ stress response to metabolic flux limitation. Mol Syst Biol 12:882. doi:10.15252/msb.20166998

Rocker A, Meinhart A (2016) Type II toxin: antitoxin systems. More than small selfish entities? Curr Genet 62:287-290

Shan Y, Brown Gandt A, Rowe SE et al (2017) ATP-dependent persister formation in Escherichia coli. MBio 8:e02267-16. doi: $10.1128 / \mathrm{mBio}$

Sturm A, Dworkin J (2015) Phenotypic diversity as a mechanism to exit cellular dormancy. Curr Biol 25:2272-2277. doi:10.1016/j. cub.2015.07.018

Unoson C, Wagner EGH (2008) A small SOS-induced toxin is targeted against the inner membrane in Escherichia coli. Mol Microbiol 70:258-270. doi:10.1111/j.1365-2958.2008.06416.x

Veening J-W, Smits WK, Kuipers OP (2008) Bistability, epigenetics, and bet-hedging in bacteria. Annu Rev Microbiol 62:193-210. doi:10.1146/annurev.micro.62.081307.163002

Vega NM, Allison KR, Khalil AS, Collins JJ (2012) Signaling-mediated bacterial persister formation. Nat Chem Biol 8:431-433. doi:10.1038/nchembio.915

Verstraeten N, Knapen WJ, Kint CI et al (2015) Obg and membrane depolarization are part of a microbial bet-hedging strategy that leads to antibiotic tolerance. Mol Cell 59:9-21. doi:10.1016/j. molcel.2015.05.011

Wang X, Wood TK (2011) Toxin-antitoxin systems influence biofilm and persister cell formation and the general stress response. Appl Environ Microbiol 77:5577-5583. doi:10.1128/AEM.05068-11

Weaver KE, Tritle DJ (1994) Identification and characterization of an Enterococcus faecalis plasmid pAD1-encoded stability determinant which produces two small RNA molecules necessary for its function. Plasmid 32:168-181. doi:10.1006/plas.1994.1053

Weel-Sneve R, Kristiansen KI, Odsbu I et al (2013) Single transmembrane peptide DinQ modulates membrane-dependent activities. PLoS Genet 9:e1003260. doi:10.1371/journal.pgen.1003260

Wen J, Won D, Fozo EM (2014) The ZorO-OrzO type I toxin-antitoxin locus: repression by the OrzO antitoxin. Nucleic Acids Res 42:1930-1946. doi:10.1093/nar/gkt1018

Wen J, Harp JR, Fozo EM (2016) The 5' UTR of the type I toxin ZorO can both inhibit and enhance translation. Nucleic Acids Res. doi:10.1093/nar/gkw1172 